PROCEEDINGS OF THE

AMERICAN MATHEMATICAL SOCIETY

Volume 140, Number 7, July 2012, Pages 2209-2214

S 0002-9939(2011)11085-2

Article electronically published on December 27, 2011

\title{
THE STRUCTURE OF FREE AUTOMORPHIC MOUFANG LOOPS
}

\author{
A. GRISHKOV, P. PLAUMANN, AND L. SABININA
}

(Communicated by Jonathan I. Hall)

\begin{abstract}
We describe the structure of a free loop of rank $n$ in the variety of automorphic Moufang loops as a subdirect product of a free group and a free commutative Moufang loop, both of rank $n$. In particular, the variety of automorphic Moufang loops is the join of the variety of groups and the variety of commutative Moufang loops.
\end{abstract}

\section{INTRODUCTION}

The variety of Moufang loops is defined by the identity

$$
(x y)(z x)=[x(y z)] x .
$$

In an arbitrary loop one defines commutators and associators by

$$
(b a)[a, b]=a b, \quad(a(b c))(a, b, c)=(a b) c .
$$

Since in a Moufang loop every subloop generated by two elements is a group, one has

$$
[a, b]=(b a)^{-1} a b, \quad(a, b, c)=(a(b c))^{-1}((a b) c) .
$$

We use the notation

$$
[Q, Q]=\langle[a, b] \mid a, b \in Q\rangle
$$

for the commutator subloop and

$$
A(Q)=\langle(a, b, c) \mid a, b, c \in Q\rangle
$$

for the associator subloop of a loop $Q$.

In an arbitrary loop $Q$ consider the translations

$$
L_{a}: x \mapsto a x, \quad R_{b}: x \mapsto x b .
$$

The group $\mathfrak{J}(Q)$ generated by the mappings

$$
l_{a, b}=L_{a} L_{b} L_{b a}^{-1}, \quad r_{a, b}=R_{a} R_{b} R_{a b}^{-1}, \quad T_{a}=R_{a} L_{a}^{-1}
$$

is called the inner mapping group of $Q$. A subloop $H$ of $Q$ is normal if $H$ is invariant under $\mathfrak{J}(Q)$ (see [3, p. 60]).

In 1956 Bruck and Paige began to study loops, in which all inner mappings are automorphisms, under the name " $A$-loops". These loops are now known as automorphic loops. In an automorphic loop $Q$ all characteristic subloops are normal, in particular, the commutator subloop $[Q, Q]$ and the associator subloop $A(Q)$ are normal subloops of $Q$.

Received by the editors October 30, 2010 and, in revised form, February 14, 2011.

2010 Mathematics Subject Classification. Primary 20N05; Secondary 20 C07.

(C)2011 American Mathematical Society Reverts to public domain 28 years from publication 
Automorphic Moufang loops form a variety which contains all groups and all commutative Moufang loops (CMLs). In [7] and [9] it is shown that every $F$ quasigroup is isotopic to an automorphic Moufang loop which is a product of a group and a CML.

In our paper we show how a free automorphic Moufang loop of rank $n$ is composed of a free group of rank $n$ and a free CML of rank $n$. In particular, the variety of automorphic Moufang loops is the join of the variety of all groups and the variety of all CMLs.

\section{Automorphic Moufang Loops}

The following result is well known (see [6, Theorem 4.16], p. 91).

Theorem 2.1. A Moufang loop $M$ is an automorphic loop if and only if the quotient loop $M / \operatorname{Nuc}(M)$ is a commutative Moufang loop of exponent 3 .

Lemma 2.2. In an automorphic Moufang loop $M$ the following identities hold:

$$
\begin{gathered}
(x, y,[z, t])=1, \\
{[(x, y, z), t]=1,} \\
(x, y, z)^{3}=1 .
\end{gathered}
$$

Proof. By Theorem 2.1 the commutator $[M, M]$ is contained in the nucleus of $M$. Hence (2.1) holds.

For $t \in M$ the mapping $x \mapsto x^{t}=t^{-1} x t$ is an automorphism of $M$. In an arbitrary loop $L$ satisfying $\operatorname{Nuc}(L) \triangleleft L$ the identities

$$
(x u, y, z)=(x, y u, z)=(x, y, z u)=(x, y, z)
$$

hold for $x, y, z \in L, u \in \operatorname{Nuc}(L)$ (see [8, Lemma 2]). For $x_{1}, x_{2}, x_{3}, t \in M$ it follows from Theorem 2.1 that $u_{i}=\left[x_{i}, t\right]=x_{i}^{-1} x_{i}^{t} \in \operatorname{Nuc}(M)$. Using equation (2.4) one obtains

$$
\left(x_{1}, x_{2}, x_{3}\right)^{t}=\left(x_{1}^{t}, x_{2}^{t}, x_{3}^{t}\right)=\left(x_{1} u_{1}, x_{2} u_{2}, x_{3} u_{3}\right)=\left(x_{1}, x_{2}, x_{3}\right) .
$$

Thus (2.2) is shown.

Identity (2.2) implies identity (i) in [3, Lemma 5.5, p. 125]. Hence $\left(x^{3}, y, z\right)=$ $(x, y, z)^{3}$ for all $x, y, z \in M$. But by Theorem 2.1] we have $x^{3} \in \operatorname{Nuc}(M)$. Hence (2.3) holds.

For an arbitrary loop $Q$ we put $D(Q)=[Q, Q] \cap A(Q)$.

Lemma 2.3. Let $M$ be an automorphic Moufang loop. Then:

(i) $D(M)$ is an elementary abelian 3-group contained in the center of $M$.

(ii) $D(M / D(M))=1$.

(iii) If $D(M)=1$, then $M$ is a subdirect product of the commutative Moufang loop $C(M)=M /[M, M]$ and the group $G(M)=M / A(M)$.

Proof. By Lemma 2.2 the identities $([x, y], z, t)=1$ and $[(x, y, z), t]=1$ hold in $M$. Hence (i) is proved, whereas (ii) and (iii) are obvious. 


\section{Free automorphic Moufang loops}

Let $\mathcal{F}_{n}$ be the free group generated by $X=\left\{x_{1}, \ldots, x_{n}\right\}$ and let $\mathcal{C}_{n}$ be the free CML generated by $Y=\left\{y_{1}, \ldots, y_{n}\right\}$. Put

$$
z_{i}=\left(x_{i}, y_{i}\right) \in \mathcal{F}_{n} \times \mathcal{C}_{n}
$$

for $1 \leq i \leq n$ and denote by $\mathcal{A}_{n}$ the subloop of $\mathcal{F}_{n} \times \mathcal{C}_{n}$ generated by $Z=$ $\left\{z_{1}, \ldots, z_{n}\right\}$. Obviously one has

Proposition 3.1. For the subloop $\mathcal{A}_{n}$ of $\mathcal{F}_{n} \times \mathcal{C}_{n}$ the following statements hold:

(i) $\mathcal{F}_{n} \times \mathcal{C}_{n}=\mathcal{A}_{n} \mathcal{C}_{n}=\mathcal{F}_{n} \mathcal{A}_{n}$,

(ii) $\left[\mathcal{A}_{n}, \mathcal{A}_{n}\right]=\left[\mathcal{F}_{n}, \mathcal{F}_{n}\right]=\left[\mathcal{F}_{n} \times \mathcal{C}_{n}, \mathcal{F}_{n} \times \mathcal{C}_{n}\right]$,

(iii) $A\left(\mathcal{A}_{n}\right)=A\left(\mathcal{C}_{n}\right)=A\left(\mathcal{F}_{n} \times \mathcal{C}_{n}\right)$,

(iv) $D\left(\mathcal{A}_{n}\right)=1$.

Lemma 3.2. Let $\mathcal{M}_{n}$ be a free automorphic Moufang loop with a base $\left\{b_{1}, \ldots, b_{n}\right\}$ and let $\varphi: \mathcal{M}_{n} \rightarrow \mathcal{A}_{n}$ be a surjective homomorphism such that $\varphi\left(b_{i}\right)=z_{i}$ for $1 \leq i \leq n$. Then $\operatorname{Ker} \varphi=D\left(\mathcal{M}_{n}\right)$.

Proof. Since $D\left(\mathcal{A}_{n}\right)=1$ it follows that $D\left(\mathcal{M}_{n}\right) \leq \operatorname{Ker} \varphi$. Conversely, we consider the restrictions

$$
\varphi_{c}:\left[\mathcal{M}_{n}, \mathcal{M}_{n}\right] \rightarrow\left[\mathcal{A}_{n}, \mathcal{A}_{n}\right], \quad \varphi_{a}: A\left(\mathcal{M}_{n}\right) \rightarrow A\left(\mathcal{A}_{n}\right)
$$

and the surjective homomorphisms

$$
\varphi^{c}: \mathcal{M}_{n} /\left[\mathcal{M}_{n}, \mathcal{M}_{n}\right] \rightarrow \mathcal{A}_{n} /\left[\mathcal{A}_{n}, \mathcal{A}_{n}\right], \quad \varphi^{a}: \mathcal{M}_{n} / A\left(\mathcal{M}_{n}\right) \rightarrow \mathcal{A}_{n} / A\left(\mathcal{A}_{n}\right)
$$

induced by $\varphi$.

Since commutators and associators form verbal subloops, the homomorphisms $\varphi_{c}$ and $\varphi_{a}$ are surjective. Consider the commutative diagram

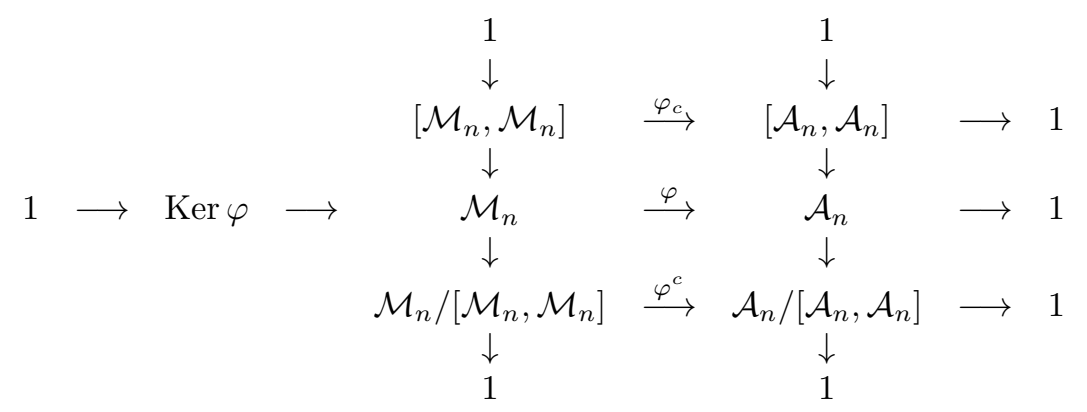

Both $\mathcal{A}_{n} /\left[\mathcal{A}_{n}, \mathcal{A}_{n}\right]$ and $\mathcal{M}_{n} /\left[\mathcal{M}_{n}, \mathcal{M}_{n}\right]$ are free CMLs of rank $n$. Hence $\varphi^{c}$ is an isomorphism. Assume that there is $w \in \operatorname{Ker} \varphi$ such that $w \notin\left[\mathcal{M}_{n}, \mathcal{M}_{n}\right]$. Considering monomorphisms as inclusions and writing surjective homomorphisms as coset projections, we obtain

$$
1=\varphi(w) \mathcal{A}_{n} /\left[\mathcal{A}_{n}, \mathcal{A}_{n}\right]=\varphi^{c}\left(w\left[\mathcal{M}_{n}, \mathcal{M}_{n}\right]\right) \neq 1,
$$

a contradiction. It follows that $\operatorname{Ker} \varphi \leq\left[\mathcal{M}_{n}, \mathcal{M}_{n}\right]$. The same argument applied to $\varphi_{a}$ gives us $\operatorname{Ker} \varphi \leq A\left(\mathcal{M}_{n}\right)$. Hence

$$
\operatorname{Ker} \varphi \leq\left[\mathcal{M}_{n}, \mathcal{M}_{n}\right] \cap A\left(\mathcal{M}_{n}\right)=D\left(\mathcal{M}_{n}\right) ;
$$

the lemma is proved. 
Theorem 3.3. The loop $\mathcal{A}_{n}$ is a free automorphic Moufang loop of rank $n$.

Proof. Let $\mathcal{M}_{n}$ a free automorphic Moufang loop with basis $\left\{b_{1}, \ldots, b_{n}\right\}$. Then there is a surjective homomorphism $\varphi: \mathcal{M}_{n} \rightarrow \mathcal{A}_{n}$ such that $\varphi\left(b_{i}\right)=z_{i}$ for $1 \leq i \leq$ $n$. We have to show that $\varphi$ is an isomorphism. In view of Lemma 3.2 this means that we have to show that $D\left(\mathcal{M}_{n}\right)=1$.

1) We first show the validity of the theorem for the case $n=3$. Let $x, y, z$ be a free generating system of $\mathcal{M}_{3}$ and let $a$ be the associator $(x, y, z)$. Then $a^{3}=1$ and $\mathbb{Z}_{3} \cong\langle a\rangle=A\left(\mathcal{M}_{3}\right)$. Assume that $D\left(\mathcal{M}_{3}\right) \neq 1$. Then $D\left(\mathcal{M}_{3}\right)=A\left(\mathcal{M}_{3}\right)$. Thus

$$
\varphi\left(\mathcal{M}_{3}\right) \cong \mathcal{M}_{3} / D\left(\mathcal{M}_{3}\right) \cong \mathcal{A}_{3}
$$

is a group, a contradiction. It follows that $D\left(\mathcal{M}_{3}\right)=1$ and $\mathcal{M}_{3} \cong \mathcal{A}_{3}$.

It is a consequence of this isomorphism that any commutator identity in at most three variables holds in all automorphic Moufang loops if and only if it holds in all groups.

In particular, the Witt identity

$$
\left[\left[x, y^{-1}\right], z\right]^{y}\left[\left[y, z^{-1}\right], x\right]^{z}\left[\left[z, x^{-1}\right], y\right]^{x}=1
$$

holds in every automorphic Moufang loop. Let $A$ be an automorphic Moufang loop such that $[A, A]$ is commutative. Then it follows from (3.1) that in $A$ the Jacobi identity

$$
[[x, y], z][[y, z], x][[z, x], y]=1
$$

is satisfied (see [10, Chap. II, $\S 6$, equation (17), p. 63]).

2) We have $\varphi\left[\mathcal{M}_{n}, \mathcal{M}_{n}\right]=\left[\mathcal{A}_{n}, \mathcal{A}_{n}\right]=\left[\mathcal{F}_{n}, \mathcal{F}_{n}\right]$ (Proposition [3.1(ii)). But $\left[\mathcal{F}_{n}, \mathcal{F}_{n}\right]$ is a free group and $\left[\mathcal{M}_{n}, \mathcal{M}_{n}\right]$ is a group. By Lemma 3.2 one $\operatorname{has} \operatorname{Ker} \varphi=$ $D\left(\mathcal{M}_{n}\right) \leq\left[\mathcal{M}_{n}, \mathcal{M}_{n}\right]$. Hence $\left[\mathcal{M}_{n}, \mathcal{M}_{n}\right]$ is the semi-direct product of $D\left(\mathcal{M}_{n}\right)$ and a subgroup $M_{c} \cong\left[\mathcal{F}_{n}, \mathcal{F}_{n}\right]$. Since $D\left(\mathcal{M}_{n}\right)$ is contained in the centre of $\mathcal{M}_{n}$ it follows that $\left[\mathcal{M}_{n}, \mathcal{M}_{n}\right]$ is even the direct product of $M_{c}$ and $D\left(\mathcal{M}_{n}\right)$.

3) By 2) we have $\left[\left[\mathcal{M}_{n}, \mathcal{M}_{n}\right],\left[\mathcal{M}_{n}, \mathcal{M}_{n}\right]\right]=\left[M_{c}, M_{c}\right]$ since $D\left(\mathcal{M}_{n}\right)$ lies in the center of $\mathcal{M}_{n}$. In particular, $\left[M_{c}, M_{c}\right]$ is a normal subloop of $\mathcal{M}_{n}$. For the quotient loop $\overline{\mathcal{M}_{n}}=\mathcal{M}_{n} /\left[M_{c}, M_{c}\right]$ and the canonical projection $\rho: \mathcal{M}_{n} \rightarrow \overline{\mathcal{M}_{n}}$, the following assertions hold:

(3.1) $D\left(\overline{\mathcal{M}_{n}}\right)=\overline{D\left(\mathcal{M}_{n}\right)} \cong D\left(\mathcal{M}_{n}\right)$.

(3.2) The abelian group $G=\left[\overline{\mathcal{M}_{n}}, \overline{\mathcal{M}_{n}}\right]$ is the direct product of the free abelian group $V=\overline{M_{c}}$ and the elementary abelian 3-group $D=D\left(\overline{\mathcal{M}_{n}}\right)$.

(3.3) $\varphi: \mathcal{M}_{n} \rightarrow \mathcal{A}_{n}$ induces a surjective homomorphism

$$
\bar{\varphi}: G \rightarrow \mathcal{F}_{n} /\left[\left[\mathcal{F}_{n}, \mathcal{F}_{n}\right],\left[\mathcal{F}_{n}, \mathcal{F}_{n}\right]\right]
$$

and $\operatorname{Ker}(\bar{\varphi})=D\left(\overline{\mathcal{M}_{n}}\right)$.

The action under conjugation of $\overline{\mathcal{M}_{n}}$ on $G$ leads to a homomorphism

$$
\gamma: \overline{\mathcal{M}_{n}} \rightarrow \text { Aut } G \text {. }
$$

The abelian group $\Gamma=\gamma\left(\overline{\mathcal{M}_{n}}\right)$ acts on $G$ such that for all $d \in D, \alpha \in \Gamma$ one has $d^{\alpha}=d$. Observe that with the notation $\gamma_{i}=\gamma\left(\overline{b_{i}}\right)$ we see that $\Gamma$ is a free abelian group generated by the set $\left\{\gamma_{1}, \ldots, \gamma_{n}\right\}$. 
4) We consider $G$ as a $\Gamma$-module and write $G$ additively for this purpose. Consider the generators $\overline{b_{i}}, 1 \leq i \leq n$ of $\overline{\mathcal{M}_{n}}$ and the elements $c_{i, j}=\left[\overline{b_{i}}, \overline{b_{j}}\right] \in G$. Obviously one has $c_{i, i}=0, c_{j, i}=-c_{i, j}$. Furthermore, $c_{i, j}, 1 \leq i<j \leq n$, forms a generating system of the $\Gamma$-module $G$.

By the Witt identity (3.2) we have for any $i, j, k$ :

$$
c_{i, j}^{1-\gamma_{k}}+c_{j, k}^{1-\gamma_{i}}+c_{k, i}^{1-\gamma_{j}}=0 .
$$

5) Now we compare the $\Gamma$-module $G$ with the standard relation module of the free abelian group $\Gamma \cong \mathbb{Z}^{n}$ (see [1], [2], [5]) and we obtain the abelian group $U=\left[\mathcal{F}_{n}, \mathcal{F}_{n}\right] /\left[\left[\mathcal{F}_{n}, \mathcal{F}_{n}\right],\left[\mathcal{F}_{n}, \mathcal{F}_{n}\right]\right]$ on which $\Gamma$ acts by conjugation, too. For the basis $x_{1}, \ldots, x_{n}$ of $\mathcal{F}_{n}$ and for $g_{i}=x_{i}\left[\left[\mathcal{F}_{n}, \mathcal{F}_{n}\right],\left[\mathcal{F}_{n}, \mathcal{F}_{n}\right]\right], 1 \leq i \leq n$, the elements $\left[g_{i}, g_{j}\right], 1 \leq i<j \leq n$, form a system of generators of $U$ as a $\Gamma$-module. Furthermore, $\bar{\varphi}: G \rightarrow U$ is an epimorphism of $\Gamma$-modules satifying $\bar{\varphi}\left(c_{i, j}\right)=\left[g_{i}, g_{j}\right]$.

By Bachmuth's Theorem [1] (see also [2]) the relations

$$
\left[g_{i}, g_{j}\right]^{1-\gamma_{k}}+\left[g_{j}, g_{k}\right]^{1-\gamma_{i}}+\left[g_{k}, g_{i}\right]^{1-\gamma_{j}}=0
$$

form a complete system of relations for $U$.

It follows from (3.3) and (3.4) that there exists a $\Gamma$-homomorphism $\tau: U \rightarrow G$ satisfying $\tau\left(g_{i, j}\right)=c_{i, j}$. Hence $\bar{\varphi} \tau=\mathrm{id}_{U}$. By 4) we know that $G=\left\langle c_{i, j}\right| 1 \leq$ $i<j \leq n\rangle^{\Gamma}$. Hence $\tau$ is a surjective and $\bar{\varphi}: G \rightarrow U$ is an isomorphism. Thus $D\left(\mathcal{M}_{n}\right) \cong D\left(\overline{\mathcal{M}_{n}}\right)=\operatorname{ker}(\bar{\varphi})=1$.

\section{ACKNOWLEDGEMENTS}

The authors acknowledge support from the programs Cátedras Especiales and Conferencias de Alto Nivel of the SRE, México, SEP-PROMEP, México, and CONACyT. Their gratitude goes to the Departamento de Cooperación y Desarroyo Internacional of the UAEM, Cuernavaca. Without the help of this institution their joint work could not have been done.

\section{REFERENCES}

[1] S. Bachmuth, Automorphisms of free metabelian groups. Trans. Amer. Math. Soc. 118, 93-104 (1965). MR0180597 (31:4831)

[2] K. A. Brown, The derived subgroup of a free metabelian group. Arch. Math. (Basel) 32, no. 6, 526-529 (1979). MR550316 (81a:20033)

[3] R. B. Bruck, A Survey of Binary Systems. Ergebnisse der Mathematik und ihrer Grenzgebiete. Neue Folge. Heft 20. Springer-Verlag, 1958. MR0093552 (20:76)

[4] R. B. Bruck, L. J. Paige, Loops whose inner mappings are automorphims. Ann. Math. 63, 308-323 (1956). MR0076779 (17:943b)

[5] R. Mikhailov, I. B. S. PAssi, Lower Central and Dimension Series of Groups. Lecture Notes in Mathematics, vol. 1952. Springer, 2009. MR2460089 (2009m:20045)

[6] H. O. Pflugfelder, Quasigroups and Loops: Introduction. Sigma Series in Pure Mathematics, 7. Heldermann Verlag, 1990. MR.1125767 (93g:20132)

[7] T. Kepka, M. Kinyon, J. D. Phillips, The structure of F-quasigroups. J. Algebra 317, no. 2, 435-461 (2007). MR2362925 (2008h:20100)

[8] P. Plaumann, L. Sabinina, On nuclearly nilpotent loops of finite exponent. Comm. Algebra 36, no. 4, 1346-1353 (2008). MR2406589 (2009c:20124) 
[9] V. Shcherbacov, On the structure of left and right F-, SM-, and E-quasigroups. Journal of Generalized Lie Theory and Applications 3, No. 3, 197-259 (2009). MR2534025|(2010k:20119)

[10] H. Zassenhaus, The Theory of Groups. Chelsea Publishing Company, 1949. MR.0030947 $(11: 77 d)$

Departamento de Matemática, Universidade de São Paulo, Caixa Postal 66281, SÃo PAUlO-SP, 05311-970, BRAZIL

E-mail address: grishkov@ime.usp.br

Department Mathematik, Universität Erlangen-Nürnberg, Cauerstrasse 11, D-90158 ERLANGEn, Germany

E-mail address: peter.plaumann@mi.uni-erlangen.de

Facultad de Ciencias, Universidad Autónoma del Estado de Morelos, Avenida Universidad 1001, 62209 Cuernavaca, Morelos, Mexico

E-mail address: liudmila@uaem.mx 